\title{
NUTRITION
}

\section{Vitamin D and Hypertension: An Overview of Current Scenario}

PK Sasidharan

\begin{abstract}
India has become the capital of all communicable diseases and noncommunicable diseases like hypertension and diabetes. Vitamin D deficiency is also very high in the Indian subcontinent (as high as $80-90 \%$ ). Therefore, it is possible that these issues are interrelated, or we should look for possible relations. The landmark study by us suggested the cause of vitamin D deficiency as poor dietary intake or in other words due to malnutrition. Vitamin D deficiency is multifactorial in etiology; the etiological factors vary from place to place and person to person. The three primary reasons for the deficiency are: lack of balanced diet, reduced sunlight exposure and increased melanin in the skin. Even if, by chance, someone gets adequate vitamin $\mathrm{D}$ from diet and sunlight, its activation in the liver and kidneys is defective due to increasing prevalence of liver and kidney diseases. Liver diseases are on the increase due to overeating with consequent obesity and nonalcoholic liver disease, or due to alcohol intake and exposure to hepatotoxic agents or infections. Subclinical liver disease is now widely prevalent in the people with consequent poor activation of vitamin D. Increasing renal diseases due to several reasons and the lack of vegetables and fruits consumption with consequent hypomagnesemia contribute to poor 1-hydroxylation in the kidneys. Lack of intake of balanced diet, lack of outdoor exercises and eating more food are all lifestyle issues, and the same are the causes for diseases, like hypertension, diabetes, ischemic heart disease (IHD), strokes and cancers and, therefore, the link between vitamin $\mathrm{D}$ deficiency and these diseases become very obvious. It is only natural that we see vitamin $D$ deficiency in all the diseases, or supplementation of vitamin $D$ benefits all the patients. To tackle the problem of vitamin $D$ deficiency in India, we need to address all the healthcare issues simultaneously and empower the people for regular intake of balanced diet, doing regular exercise and exposure to sunlight through systematic and meticulous social, educational, agricultural and financial reforms. Therefore, all disease control programs in India should focus on empowering the people, by the necessary social reforms and its implementation. As an interim measure, fortification of foodstuffs like oil or wheat-flour also may be tried till such time that all sections of the society are empowered for taking balanced diet regularly.
\end{abstract}

Keywords: Balanced diet, Consumerism, Hypertension, Lifestyle, Vitamin D.

Senior Consultant Physician

Department of Medicine, PVS Hospital, Calicut, Kerala, India

Corresponding Author: PK Sasidharan, Senior Consultant Physician, Department of Medicine, PVS Hospital, Railway Station Road, Calicut, Kerala, India, Phone: 9847005177 , e-mail: sasidharanpk@gmail.com
How to cite this article: Sasidharan PK. Vitamin D and Hypertension: An Overview of Current Scenario. Hypertens J 2015;1(1):34-39.

Source of support: Nil

\section{Conflict of interest: None}

\section{INTRODUCTION}

Hypertension (HTN) is a man-made disaster, like diabetes and all other killer diseases. The name essential hypertension wrongly suggests that we are not responsible for its development; it is not essential to have hypertension, it is preventable. We need to see what is hypertension and why the incidence of hypertension is increasing all over the globe, what is the status of vitamin D in hypertensives, how hypertension and vitamin D are related, and why vitamin $\mathrm{D}$ deficiency and hypertension are becoming a global issue and what are the solutions.

\section{ETIOLOGY OF ESSENTIAL HYPERTENSION}

The normal blood pressure is generated in the circulation by the pumping action of heart (cardiac output) and the resistance offered by the blood vessels (peripheral resistance). There are numerous determinants and variables, which influence both cardiac output and peripheral resistance. High blood pressure is only the end result of several adjustments the body makes in response to small-unnoticed disturbances in one or more determinants of cardiac output and peripheral resistance. When such disturbances happen regularly which tend to increase either cardiac output or peripheral resistance or both over several years, and when these disturbances are allowed to go on without any break, in the end the high blood pressure becomes persistent and is called essential hypertension.

When any one of the numerous factors influencing normal blood pressure is upset to the extremes, it alone can manifest as hypertension, we call it as secondary hypertension. Thus, secondary hypertension is a situation where high blood pressure is due to a single identifiable etiology, the correction of which may lead to regression of blood pressure. Each secondary hypertension gives us insight into the mechanisms of essential hypertension. If we have insight into the factors which influence the normal blood pressure and all the causes of secondary hypertension, we can derive the etiology of essential 
hyperextension too. It is because essential hypertension is due to a combination of several of these factors, which influence the normal blood pressure, when they come together accidentally or incidentally over a long period in the life of a person. Even in the secondary hypertension, several other factors as in the case of essential hypertension may be operating in addition, and they have to be identified and corrected for optimal benefit to the patient. The combination of factors, which are upset in each patient, in essential hypertension varies from person to person. Therefore, each patient with hypertension is different and we need to manage him or her with that mindset to give a highly individualized treatment for better results.

\section{What is the Current Status of Essential Hypertension?}

Why is essential hypertension becoming an epidemic? Decades back, people used to engage in physical activity to make a living, they were eating less, and consumed food from natural sources, they were not exposed to excess salt or toxins from packaged foods or fast foods and to so much stress; thus, there was no hypertension and diabetes. Of course, some people with aberrancies would have developed it, but that was a rarity. Over the decades, the lifestyles and food habits have changed. All these changes can influence the determinants and variables of blood pressure, all under the influence of the consumerist forces-increased caloric intake, that too the fast foods and junk foods with excess of salt and calories, reduced exercise and sedentary lifestyle; all of these promoted by the advertisements in the media using the celebrities, including those who conduct cookery shows. Consequent to the unwanted influences and the lack of appropriate empowerment, there is overeating, increasing sedentary habits, increased salt consumption, reduced water intake, low intake of vegetables and fruits, undue stress and anxiety. Even in the name of fruits and vegetables, some people end up eating only the fast foods; because of several such issues there is deficiency of several micronutrients and vitamins. When such a situation is combined with bad habits like smoking and alcohol, and when the consumerist forces influence them to purchase or consume unnecessarily, it pushes them into anxiety, depression and debt traps. All these, together, have given us all the non-communicable diseases as a package, and thus India has ended up as the disease capital of the world. All this is because of lack of empowerment, education, improper economic and social reforms resulting in dangerously inappropriate distribution of wealth, corruption and black money, one encouraging the other. It is time for a change for the benefit of the next generation. The more we consume in any form, the more we are likely to become hypertensive, poorer the quality of food higher are the chances of becoming hypertensive, the more stressed we are higher is the risk of developing hypertension.

\section{Vitamin D}

Vitamin D belongs to the class of vitamins, but it is unique because it is also synthesized in the body and functions as a hormone. It has crucial roles in calcium homeostasis, bone mineral metabolism, cellular growth and differentiation and immunomodulation. It is also an important effecter of macrophage functions and plays a significant role in limiting growth or survival of intracellular pathogens, like Mycobacterium tuberculosis, Salmonella and viruses. ${ }^{1-6,14}$ Recent evidences link vitamin D deficiency to all diseases including infections, diabetes, hypertension, autoimmune disorders, cancer and dyslipidemia. ${ }^{8,9,15}$ Vitamin D modifies gene expression by binding to specific receptors [vitamin $D$ receptor (VDR)] in the tissues where it acts, and its actions are not fully known to us at the moment. ${ }^{2-4,7,8}$

There are two natural sources of Vitamin D; one is through biosynthesis in skin and the other through diet. ${ }^{7}$ Cutaneous biosynthesis is under the influence of ultraviolet light and is possible only if we get adequate sunlight exposure without too much of obstructive clothing. High melanin content and obstructive clothing could be interfering with cutaneous synthesis. The dietary sources are pulses (for a vegetarian diet) and egg, fish and meat (for nonvegetarians); but is present in adequate amounts only in a well balanced diet. Whatever may be the source, it has to be activated in the liver by a 25 -hydroxylation and in the kidneys by $\alpha$-hydroxylation, to produce the active 1,25 dihydroxy vitamin $\mathrm{D}[1,25-(\mathrm{OH}) 2 \mathrm{D}] .{ }^{79}$ The daily dietary requirement is around 400 to $1000 \mathrm{IU}$, previously taken as $200 \mathrm{IU} .79$ There was no consensus on the normal levels until recently, and now the accepted normal serum level is 30 to $60 \mathrm{ng} / \mathrm{ml} .{ }^{9}$ Insufficiency is said to occur when the levels are less than $30 \mathrm{ng} / \mathrm{ml}$ and deficiency at less than $20 \mathrm{ng} /$ $\mathrm{ml} .^{7,9,11,12}$ Being a tropical country, it was firmly believed that vitamin D deficiency did not exist in India which was first disproved by a landmark study, and the causes for the deficiency was indicated as malnutrition in the same article but is still not widely known or accepted. ${ }^{1}$

\section{Vitamin D and Hypertension}

It is known that VDRs are present in vascular smooth muscle, endothelium and heart. Therefore, it is natural that it must have some useful action on these organs, whereby it could modify blood pressure to the advantage 
of the body. It is also now observed that vitamin D inhibits renin expression in the juxtaglomerular apparatus, therefore, the deficiency could activate renin-angiotensinaldosterone system (RAAS). ${ }^{17}$ Thus, it appears that vitamin $\mathrm{D}$ deficiency can increase peripheral resistance. The active 1,25-(OH)2D could prevent foam cell formation by reducing modified low-density lipoprotein (LDL) cholesterol uptake in macrophages, therefore, it could prevent atherosclerosis. It is also known that the commonest cause for reno-vascular hypertension is atherosclerotic narrowing of the renal arteries and it could also be a link to HTN and vitamin D. ${ }^{2,10,15}$

\section{Vitamin D Status in Indian Patients with Hypertension}

The original study by us has given a picture of vitamin $\mathrm{D}$ status in hypertension. ${ }^{16}$ The study was undertaken since the role of hypovitaminosis $\mathrm{D}$ in lifestyle diseases has not been well studied in India. There were 55 subjects and 32 controls, whose 25 hydroxy vitamin D [25- $(\mathrm{OH})$ D] levels, daily sunlight exposure, dietary intake, and physical activity were assessed. The mean $25-(\mathrm{OH}) \mathrm{D}$ level for hypertensives was found to be $17.07 \pm 9.72 \mathrm{ng} / \mathrm{ml}$, while in the normotensive controls was $22 \pm 10 \mathrm{ng} / \mathrm{ml}(\mathrm{p}=0.027)$. Only $20 \%$ of the total population, i.e. cases and controls together, had sufficient levels above $30 \mathrm{ng} / \mathrm{ml}$. Higher dietary intake, i.e. > $200 \mathrm{IU} /$ day were, found to have lesser incidence of HTN. Conclusion of the study were that vitamin D levels are significantly lower in the hypertensive population as compared to normotensives. In addition, overall the 25-(OH)D levels are significantly low in both cases and controls. Adequate dietary intake of vitamin D has a lesser chance of developing hypertension. Adequate sunlight exposure and physical activity are protective against hypertension. ${ }^{16}$

\section{The Social Aspects of Vitamin D Deficiency: How is it related to Lifestyle and Hypertension? What are the Solutions?}

The reasons for the widespread deficiency of vitamin $\mathrm{D}$ in India were indicated in the landmark study as lack of balanced diet, reduced sunlight exposure and increased melanin in the skin. ${ }^{1}$ On closer observation of the problem for almost three decades, it became clearer that these root causes are the result of several issues and all are interlinked with diet and lifestyle habits.

\section{What is Good Lifestyle?}

Good lifestyle include several habits like-eating a balanced diet daily, drinking adequate water every day, avoiding tobacco smoking and alcohol, overeating, fried food and fast food, etc. Good lifestyle also involves doing some kind of physical exercise regularly, avoiding undue mental stress, adopting the right posture while working, sitting, travelling or while using computers, and even the practice of good waste disposal habits. It also involves ensuring safety of food and water, oral hygiene, hand washing and respiratory hygiene. In a broader sense, it is the absence of good lifestyle practices that lead to diseases, like infections, hypertension, diabetes, heart attacks, strokes, obesity and cancers. The research tools that we employ now are not enough to find out root causes of such diseases; but the root causes can always be found out, by closer observation and comparison of the diet, lifestyle and environment of those who get diseases and of those who remain healthy without any diseases, even after 80 or 90 years. All the abnormal lifestyle practices, in turn, can be traced to lack of empowerment and lack of basic facilities, and unchecked and unhealthy promotion of consumerism. The root causes of all diseases, thus lie in diet, lifestyle and environment we live in, and all are linked to the social issues around us. The observations on the causes of vitamin D deficiency in relation to lifestyle are given below.

\section{Causes of Vitamin D Deficiency}

1. Lack of regular consumption of balanced diet is the commonest cause of vitamin D deficiency-it is the conclusion based on three decades of close observation by the author. Proper diet and adequate sunlight exposure would provide the inactive form of vitamin D, but more important is balanced diet. It is not an exaggeration if we say our people eat a balanced diet only by chance or by accident and not by plan. Majority of the people in India do not even have the chance to get a balanced diet even by accident due to the lack of social empowerment. The truth is that those who are likely to get balanced diet never get any sunlight exposure and those who get enough sunlight never get balanced diet. The practice of taking a balanced diet every time while eating is the most important good lifestyle habit, and the society is not empowered for it in India now, though we have the resources. Majority of the people are in dark about the concept of a balanced diet, and even if they if they have any, they are often wrong. Balanced diet should contain one source of calories (e.g. any one cereal in the Indian diet, no cereal is superior in that), adequate protein (any one of the pulses/curd/ fish/egg or meat), adequate vegetables-preferably raw or steamed only and never overcooked, fresh whole seasonal fruits, and adequate safe drinking water. Therefore, vegetarians should always eat a 
combination of 'any one cereal + any one of the pulses + vegetables + fruits + water' in the right proportions, every time we eat (nonvegetarians should take egg, fish or meat instead of the pulses). If we are using roots and tubers as a source of calories, as in a western diet, the source of protein should always be nonvegetarian or at least curd should be used as a source of protein. Lack of awareness about balanced diet, some beliefs and wrong concepts about diet and lack of social empowerment prevent many from taking dietary items, which contain vitamin D. In Kerala, for example, most people avoid pulses believing that it produces gas or dyspepsia, even as their diet does not contain nonvegetarian items as a source of protein. The gas or dyspepsia is due to disordered motility of the gastrointestinal tract due to reduced fiber in the diet resulting from decreased intake of vegetables and fruits, which are also necessary for adequacy of vitamin $D$, as described later. There are only very few people who regularly get all these in their diet. The reasons for the poor intake of vitamin $\mathrm{D}$ containing food items is a complex issue with social, financial, religious and cultural implications. Even affluent people do not consume a balanced diet, but consume fast foods and junk foods and many of them are exposed to overeating, sedentary habits, alcohol and tobacco smoking. Even in the diets of affluent people, adequate amounts of protein is missing; but some rich people end up eating excess protein with development of hyperuricemia which, in turn, can damage the kidneys. The practice of taking vegetables and fruits regularly, is also important for vitamin D metabolism, but is notoriously absent even among the affluent people. Balanced diet should include adequate water intake also. Inadequate water intake and high salt intake are wrong dietary habits which can activate the mechanisms for increasing the blood pressure and cause damage to the kidneys. Even provision for safe drinking water to all citizens is lacking in our country. Urgent attention from all quarters, to empower the society for regular consumption of balanced diet, is absolutely essential to solve the problem of vitamin D deficiency and for fighting all diseases, because 'food is the primary medicine' ${ }^{18}$

2. Hypomagnesemia is an important reason for vitamin $\mathrm{D}$ deficiency. It primarily results from poor intake of vegetables and fruits, besides renal and gastrointestinal losses due to multiple reasons. The 1-hydroxylation of vitamin $\mathrm{D}$ in the kidneys is parathyroid hormone (PTH) dependent, and PTH secretion in turn is magnesium dependent. Besides reduced intake of vegetables and fruits and renal losses, there is poor absorption of magnesium from the intestine due to parasites and infections like tuberculosis of intestine. Thus, an individual could have multiple reasons for hypomagnesemia, which is always subclinical and is often overlooked. Regular and adequate intake of vegetables and fruits is an important lifestyle habit, which is absent in the majority of people all over the world. Besides magnesium, vegetables and fruits provide potassium, fiber, folic acid, antioxidants and even digestive enzymes. Thus, magnesium deficiency due to multiple causes, would lead to reduced PTH secretion; and the consequent reduction of 1-hydroxylation of vitamin D in the kidneys is an important and unrecognized cause of vitamin D deficiency. $7,12,13$

3. Third largest issue behind vitamin D deficiency is defective 25-hydroxylation of dietary or cutaneously synthesized vitamin D, due to clinical and subclinical liver diseases. The incidence and prevalence of liver diseases is steadily increasing every year. Among the relatively affluent and the rich people, overeating and the consequently increased body mass index (BMI), and development of fatty liver and chronic liver diseases are the commonest reasons for liver disease. But that is not standing alone; the same people are also exposed to alcohol and other toxins from the fast foods, junk foods and the fried foods, and their diet is devoid of protective items because they usually do not eat vegetables and fruits adequately. The growing acceptance of alcohol with moderate to heavy intake, and exposure to other hepatotoxic substances through diet and as medicines and health preparations (including indigenous medicine) is common among all groups including the poorer ones. Most of the issues in liver diseases, thus, are related to lifestyle practices and unchecked consumerism; especially when it comes to overeating, lack of physical activity and exposure to hepatotoxic substances. The same issues behind nonalcoholic fatty liver diseases are the causes for essential hypertension too. In addition, large number of people get infective hepatitis and survive and live with subclinical liver disease.

4. Another important cause for the deficiency is defective metabolism of vitamin D in the kidneys (the 1-hydroxylation). Kidney diseases, clinical and subclinical, due to several reasons are also increasing. Hypertension and diabetes are the leading causes of significant renal damage. In addition, renal damage is possible due to reduced water intake, frequent exposure to toxins in diet and environment, high protein diet in the affluent with consequent hyperuricemia, and nephrotoxic drugs like nonsteroidal anti-inflammatory drugs (NSAIDs) as over-the-counter medications. Toxins in the diet and environment that are potentially 
damaging to the kidneys could be cadmium, mercury, lead, and copper from electronic equipment or from some indigenous preparations. The habit of taking adequate safe water regularly is an important good lifestyle habit, which is absent in majority, including the affluent ones. Adequate water (approximately 2 liters per day) intake is absolutely essential to prevent development of high blood pressure, renal damage from hyperuricemia and urinary tract infections. In controlling high blood pressure, doctors stress only on salt restriction often ignoring the role of adequate water intake, which has several other benefits as well. Renal loss of magnesium as a result of clinical and subclinical renal damage, or due to drugs also contributes to vitamin D deficiency as mentioned before.

5. Decreased sunlight exposure: Even if there is enough sunlight in India, the habit of exposing our body to sunlight, even by accident, is becoming very rare due to changing lifestyle, lack of outdoor activities, and lack of empowerment. The preference for indoor jobs alone and not doing any outdoor activities or exercise is a lifestyle disorder resulting in reduced cutaneous biosynthesis of vitamin D. Lack of playgrounds, lack of availability or vanishing public places and parks for doing exercise is also an issue in cities now.

6. The increasing use of obstructive clothing due to imitation of the western culture or for religious reasons could be contributing to reduced sunlight exposure.

7. Even if we expose our skin to sunlight, the increased melanin content in the skin of our people, could be interfering with ultraviolet light mediated vitamin D synthesis. ${ }^{7,11}$

\section{SOCIAL SIGNIFICANCE OF THE STUDY AND THE OBSERVATIONS}

The study suggested that vitamin D deficiency is very common in the subjects, including the apparently healthy controls, which were representative sample of the people of Kerala and could indicate similar situation in the rest of the country. The author's observation spanning three decades is that vitamin D deficiency is only the tip of the iceberg of malnutrition and of wrong lifestyle habits in a society. It is malnutrition which initiates or perpetuates diseases like tuberculosis and even progression of HIV infection to AIDS, since proper nutrition and vitamin $D$ has decisive role in maintaining cell-mediated immunity. ${ }^{1-5}$ Vitamin D deficiency is only an indicator of the subclinical and clinical malnutrition, which is widely prevalent all over the country. Subclinical malnutrition is present in all diseases including hypertension and diabetes, and that is why vitamin D deficiency is seen in all diseases. All disease-control programs, in order to succeed, need to incorporate dietary intervention, education and empowerment of the people for regular intake of balanced diet and exercise in sunlight. This observation is particularly relevant since even relatively affluent section of society do not really know of or consume a balanced diet; but they consume all kinds of fast foods, junk foods, and many of them are exposed to overeating, sedentary habits, alcohol and tobacco smoking, and develop organ damages interfering with vitamin $\mathrm{D}$ metabolism. The very same people are confining to indoor activities or do white-collar jobs only with hardly any sunlight exposure. Evidence is emerging regarding the role of vitamin $\mathrm{D}$ in decreasing the risk of chronic illnesses, including common cancers, autoimmune diseases, other infectious diseases, like hypertension, diabetes and cardiovascular ones, all indicating the role of balanced diet in controlling all these diseases. ${ }^{1,8,9,11-13}$ It is reported that raising the serum 25 $(\mathrm{OH}) \mathrm{D}$ level up to 40 to $60 \mathrm{ng} / \mathrm{ml}$ would prevent breast, prostate and colorectal cancers. There are no risks from intake of 1000 to 2000 IU per day of vitamin D3. ${ }^{9-11}$ Vitamin D deficiency is also found to be associated with low high density lipoproteins, high triglyceride and high total cholesterol. ${ }^{15}$ Thus, it is obvious that all diseases are converging finally to wrong diet and lifestyle. Clinicians in India are witnessing an increase in lifestyle disorders, autoimmune disorders and malignancies. This could be because of the reason that the already malnourished people are now getting exposed to wrong lifestyle habits (thrown open by unregulated promotion of consumerism). The focus should be on empowering the people to get balanced diet regularly as part of a good lifestyle habit. Lifestyle improvement should also include facilities for doing exercise in open for adequate sunlight exposure, and for avoiding bad habits, like overeating, fast foods, fried foods, smoking and alcohol. Temporarily, there should be nationally coordinated action to substantially increase intake of vitamin D and calcium as nutritional supplements. As a long-term measure, several social, educational, agricultural, law and economic reforms are needed to solve the issues. ${ }^{18}$

\section{RECOMMENDATIONS}

There is an urgent need to provide the basic health needs and improve the dietary habits and other lifestyle habits in India by social, educational, agricultural and financial reforms rather than focusing on treatment of diseases alone. The people who engage in healthcare activities and those who are in decision-making posts should be aware of the intricate relation between social issues and diseases. All sections of people should get balanced diet and adequate exposure to sunlight. All disease control programs should focus on empowering the people for 
regular intake of balanced diet. As an interim measure, fortification of foodstuffs like oil or wheat-flour also may be tried until all sections of the society are empowered for taking balanced diet regularly. To get sunlight exposure, there must be facilities for doing physical exercise in open, like adequate play grounds, footpaths, cycle paths, parks, etc., and people should be made aware of and empowered to make use of all these. If exercise is done in natural setting, we would get enough sunlight; else, sun exposure by plan, should be included in good lifestyle habit. To conclude, all the people should be empowered to take a balanced diet and to do regular exercise as a part of their lifestyle to solve the problem of vitamin D deficiency in the society.

Several randomized clinical trials (RCTs) suggest that vitamin D supplementation may reduce blood pressure modestly in patients with hypertension, but the effects are inconsistent and subject to proper re-analysis of the data. However, since hypertension and cardiovascular diseases (CVD) may occur with greater frequency in individuals with vitamin $\mathrm{D}$ deficiency, the subject remains open for therapeutic intervention. From a public health point of view, it makes much sense for the regulators to revise the recommended daily allowance (RDA) values for calcium and vitamin D intake for all age groups. Policies involving bone and mineral health should incorporate its connectivity to cardiovascular health. What is needed urgently by the food industry is to fortify various foods and ingredients. Technical expertise is needed to fortify commonly consumed foods. Perhaps, as a pilot program, there should be mandated distribution of vitamin $D$ fortified foods in schools and colleges.

The only way to detect, prevent and treat vitamin D deficiency in the country is through a comprehensive sustained partnership between the medical community healthcare workers, social agencies, educators, consumers and the government. Future studies should examine the potential value of vitamin D supplementation with traditional antihypertensive strategies, and to evaluate the cardiovascular outcomes. We should also develop national guidelines for detecting and correcting vitamin $D$ deficiency in the community. Daily doses of vitamin D used in the studies vary widely between 300 and 2000 IU per day; we should come up with an optimal dosage required to correct vitamin $\mathrm{D}$ deficiency so that some uniformity is achieved in clinical practice. Importantly, studies are needed to establish a cause-effect relationship between vitamin D metabolism and cardiovascular health, and the underlying physiological mechanisms.

\section{REFERENCES}

1. Sasidharan PK, Rajeev E, Vijayakumari V. Tuberculosis and Vitamin D deficiency. J Asso Physic Ind 2002;50:554-558.

2. Henry HL, Norman AW. Vitamin D: Metabolism and biological actions. Annu Rev Nutr 1984;4:493-520.

3. Denis M. Killing of mycobatcerium tuberculosis within human monocytes, activation by cytokines and Calcitriol. Clin Exp Immunol 1991:84(2):200-206.

4. Abu-Amer Y, Bar-Shavit Z. Impaired bone marrow derived macrophage differentiation in vitamin $\mathrm{D}$ deficiency. Cell Immunol 1993:151(2):356-368.

5. Kreutz M, Anderson R. Induction of human monocyte to macrophage maturation in vitro by 1,25-dihydroxyvitamin D3. Blood 1990:76(15):2457-2461.

6. Crowle AJ, Salfinger M, May MH. 1,25 DH D3 synergises with pyrazinamide to kill tubercle bacilli in cultured human macrophages. Am Rev of Res Dis 1989:139(2):549-562.

7. Richard, Bringhurst F.; Marie, Demay B.; Stephen, Krane M.; et al. Harrison's Principles of Internal Medicine. 17th ed. New York: McGraw Hill; 2008. Disorders of Bone and Mineral Metabolism in Health and Disease.

8. Garland CF, Gorham ED, Mohr AR, Garland FC. Vitamin D for cancer prevention: global perspective. Ann Epidemiol 2009 Jul 1;19(7):468-483.

9. Holick MF. Vitamin D deficiency. N Engl J Med 2007;357: 266-281.

10. Scragg R, Sowers M, Bell C. Serum 25-Hydroxyvitamin D, ethnicity and blood pressure in the Third National Health and Nutrition Examination Survey. Am J Hypertens 20(7): 713-719.

11. Lips P. Vitamin D deficiency and secondary hyperparathyroidism in the elderly: Consequences for bone loss and fractures and therapeutic implications. Endocr Rev 2001 Aug;22(4):477-501.

12. Stechschulte SA, Kirsner RS, Federman DG. Vitamin D: Bone and Beyond, Rationale and Recommendations for Supplementation. Am J Med 2009 Sep;122(9):793-802.

13. Institute of Medicine (US): Standing committee on scientific evaluation of dietary reference intakes. Dietary reference intakes for Calcium, Phosphorus, Magnesium, Vitamin D and Fluoride. Washington DC: National Academy press; 1997. Available at: http://www.ncbi.nlm.nih.gov/books/ NBK109825/

14. Sasidharan PK. Tuberculous osteomyelitis and vitamin D deficiency. J Orthop 2004;1(2):e3.

15. Vitamin D levels and dyslipidemia-population-based study in Finland. J Int Med 2010 Dec;268(6):604-610.

16. Velayudhan G, Sasidharan PK. Vitamin D Status in Hypertension. Am Int J Res Formal Appl Nat Sci 2014;8(1):28-30.

17. International Journal of Endocrinology, Volume 2010(2010): 10.1155/2010/579640.

18. Sasidharan PK. Vitamin D deficiency and lifestyle diseases in India, the relation and the solutions "Progress in Medicine 2015;25 Publication of the Association of Physicians of India Chapter 27, 148-154. 